\title{
Aterectomia Rotacional para o Tratamento da Reestenose Intrastent
}

\author{
Daniel Zanuttini ${ }^{1}$, Costantino O. Costantini ${ }^{1}$, Sergio G. Tarbine ${ }^{1}$, Marcelo F. Santos ${ }^{1}$, \\ Marcos A. Denk' ${ }^{1}$ Costantino R. Costantini' ${ }^{1}$ Ademar M. de Souza ${ }^{1}$
}

\section{RESUMO}

Relatamos o caso de um paciente com reestenose de stent não-farmacológico, secundária a hipoexpansão da prótese, decorrente de grave calcificação da placa aterosclerótica subjacente, a qual não foi adequadamente reconhecida na angiografia. A utilização do ultrassom intracoronário foi de grande importância para reconhecer o mecanismo da reestenose e orientar na escolha da estratégia intervencionista mais adequada. A aterectomia rotacional foi realizada com sucesso para debilitar o cálcio peristent, permitindo a expansão posterior da prótese com balões de alta pressão.

DESCRITORES: Reestenose coronária. Calcinose. Doença da artéria coronária/ultra-sonografia. Recidiva. Ultra-sonografia de intervenção. Angioplastia transluminal percutânea coronária. Stents. Homem. Meia-idade. Relatos de casos.
0 principal mecanismo de reestenose intrastent é a hiperplasia neointimal'. O ultrassom permite reconhecer outros mecanismos relacionados a esse processo, como fratura ou hipoexpansão do stent ${ }^{2}$. A hipoexpansão pode ser consequência de escolha inadequada do diâmetro do stent em relação à referência do vaso, de expansão insuficiente do stent, apesar da seleção apropriada de seu tamanho ou de falta de conhecimento de certas características da placa (por exemplo, placas fibróticas e/ou calcificadas), que podem não permitir a expansão adequada do stent, apesar da escolha correta de seu diâmetro e de insuflações a alta pressão. O objetivo deste caso foi demonstrar a importância do ultrassom intravascular no reconhecimento do mecanismo da reestenose, assim como apresentar uma alternativa para o tratamento da

1 Hospital Cardiológico Costantini - Fundação Francisco Costantini Curitiba, PR, Brasil.

Correspondência: Daniel Zanuttini. Rua Pedro Collere, 890 - Vila

Izabel - Curitiba, PR, Brasil - CEP 80320-320

E-mail: danielzanuttini@hotmail.com

Recebido em: 18/5/2009 • Aceito em: 18/11/2009

\section{ABSTRACT}

\section{Rotational Atherectomy for the Treatment} of Instent Restenosis

We report a case of a patient with bare metal stent restenosis secondary to stent underexpansion, due to a heavily calcified atherosclerotic plaque, which was not identified by angiography. Intravascular ultrasound was important to recognize the mechanism of restenosis and determine the best strategy to be used. Rotational atherectomy was successfully used to weaken peristent calcium, allowing the posterior expansion of the prosthesis using high pressure balloon dilatation.

DESCRIPTORS: Coronary restenosis. Calcinosis. Coronary artery diseases/ultrasonography. Recurrence. Ultrasonography, interventional. Angioplasty, transluminal percutaneous coronary. Stents. Male. Middle aged. Case reports.

hipoexpansão de stents refratária à dilatação com balões de alta pressão.

\section{RELATO DE CASO}

Paciente do sexo masculino, com 57 anos de idade, deu entrada em nossa instituição em maio de 2008 para realização de cinecoronariografia. Não referia sintomas, porém apresentava ecocardiograma de estresse evidenciando isquemia no segmento médio apical da parede anterior e segmentos apicais das paredes inferior e lateral (Figura 1). Dentre os fatores de risco cardiovascular, destacavam-se história familiar de doença coronária, dislipidemia, diabetes melito, passado de tabagismo e obesidade. Referia a prática de atividade física regular, sem apresentar precordialgia ou equivalentes anginosos. Há nove meses, em outro hospital, havia tratado a artéria descendente anterior com implante de stent não-farmacológico $3 \mathrm{~mm} \times 18 \mathrm{~mm}$.

À cineangiocoronariografia realizada em nossa instituição, o segmento inicial da artéria coronária direita demonstrava lesão de $30 \%$ e o ramo descendente posterior apresentava em seu óstio lesão de 30\%. O tronco da coronária esquerda apresentava-se livre de doença aterosclerótica. A artéria descendente anterior apre- 


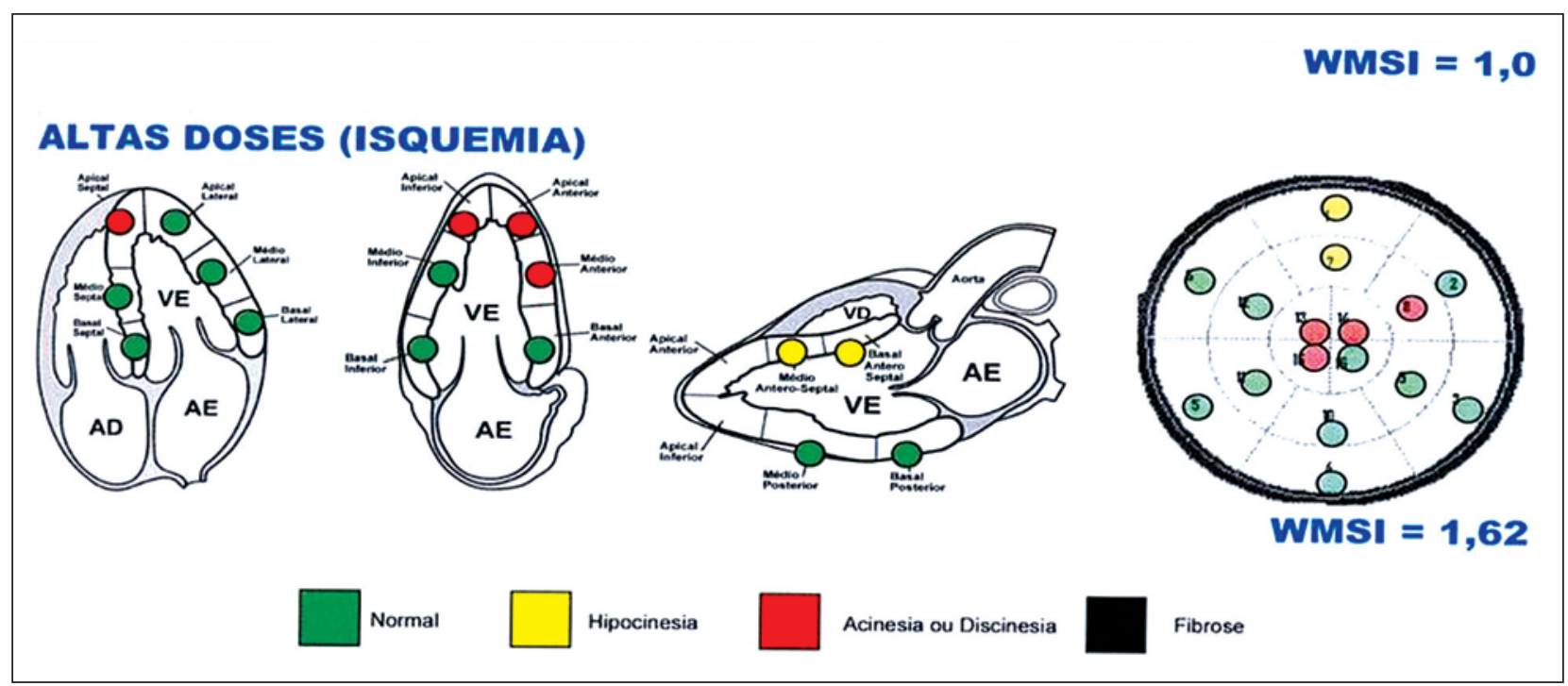

Figura 1 - Ecocardiograma de estresse demonstrando isquemia no segmento médio apical da parede anterior e segmentos apicais das paredes inferior e lateral. $\mathrm{AD}=$ átrio direito; $\mathrm{AE}=$ átrio esquerdo; $\mathrm{VD}=$ ventrículo direito; $\mathrm{VE}=$ ventrículo esquerdo.

sentava em seu terço proximal stent com reestenose suboclusiva, tipo II da classificação de Mehran ${ }^{3}$. A artéria circunflexa mostrava irregularidades parietais discretas (Figura 2) e a ventriculografia esquerda demonstrou volumes e contratilidade preservados.

O paciente foi submetido a nova intervenção percutânea, sendo realizada avaliação com ultrassom intracoronário, o qual evidenciou falta de expansão da prótese associada a hiperplasia neointimal intrastent. O segmento proximal do stent apresentava área do stent de 11,12 $\mathrm{mm}^{2}$ e área luminal de 11,03 mm²; o segmento médio, local da hipoexpansão, apresentava área do stent de 3,47 $\mathrm{mm}^{2}$ e área luminal de 3,19 m²; e o segmento distal, área do stent de 9,22 $\mathrm{mm}^{2}$ e área luminal de $8,75 \mathrm{~mm}^{2}$. Foi realizada tentativa de expansão da prótese com a utilização de cateter-balão nãocomplacente $3,5 \mathrm{~mm} \times 10 \mathrm{~mm}$ de comprimento, até 26 atm, sem sucesso. Avaliação com ultrassom intracoronário demonstrou que a área do stent não ultrapassou 3,52 $\mathrm{mm}^{2}$ e a área luminal, 3,26 $\mathrm{mm}^{2}$.

Optou-se, então, pela realização de aterectomia rotacional, com aumento progressivo do tamanho das ogivas $(1,75,2,00,2,15$ e 2,25). Posteriormente foi realizada nova tentativa de dilatação com cateter-balão $4 \mathrm{~mm} \times 11 \mathrm{~mm}$, insuflado até $26 \mathrm{~atm}$. No controle angiográfico, observou-se bom ganho luminal, com diâmetro luminal mínimo de 2,09 mm e estenose residual de $29 \%$ pela análise angiográfica quantitativa (Figura 3). Na avaliação com ultrassom intracoronário, observou-se expansão satisfatória, com área do stent de 6,12 $\mathrm{mm}^{2}$ e área luminal final de 5,33 $\mathrm{mm}^{2}$ (Figura 4).

O procedimento transcorreu sem complicações e o paciente recebeu alta 48 horas após a angioplastia. Após um ano de evolução, o paciente encontra-se assin- tomático, sem eventos cardíacos adversos e sem sinais de isquemia evidentes ao ecocardiograma de estresse realizado no seguimento clínico.

\section{DISCUSSÃO}

A calcificação faz parte da história natural da aterosclerose $^{4}$, sendo o ultrassom método muito sensível para sua detecção. A calcificação na parede coronária é classificada como superficial ou profunda, de acordo com sua localização, sendo também medida em graus (0 a 360), de acordo com sua distribuição circunferencial.

A calcificação acentuada de uma lesão aterosclerótica pode resultar em hipoexpansão do stent, mesmo após pré-dilatação com balão a alta pressão. Métodos adjuntos de ablação, como aterectomia rotacional e laser, têm sido empregados nesses casos para modificar a complacência do vaso, garantindo a expansão adequada do stent ${ }^{5-7}$.

O caso aqui relatado mostra stent implantado de forma inadequada, em decorrência de calcificação grave de placa aterosclerótica subjacente, em arco de 360 graus, a qual não foi apropriadamente diagnosticada e modificada antes do implante do stent. Essa má expansão da prótese, associada à hiperplasia neointimal, levou ao comprometimento significativo da luz do vaso e consequente isquemia miocárdica. Insuflações com cateter-balão a alta pressão foram ineficazes em fraturar o anel de cálcio e, consequentemente, em expandir o stent. A aterectomia rotacional foi realizada no segmento reestenótico, com velocidade ajustada para 150.000 rpm, utilizando-se diâmetros da ogiva progressivamente maiores até $2,25 \mathrm{~mm}$ e períodos de ablação inferiores a 30 segundos, tomando-se o cui- 


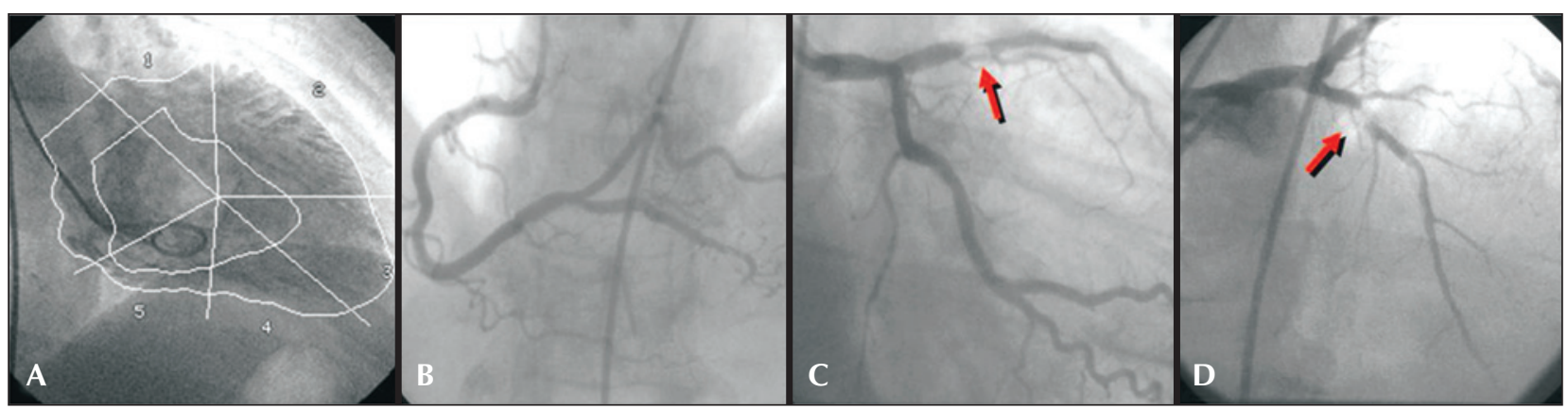

Figura 2 - Cineangiocoronariografia pré-intervenção: A) ventriculografia; B) coronária direita; C) coronária esquerda em projeção oblíqua anterior direita; D) coronária esquerda em projeção ântero-posterior cranial. As setas indicam a reestenose intrastent.

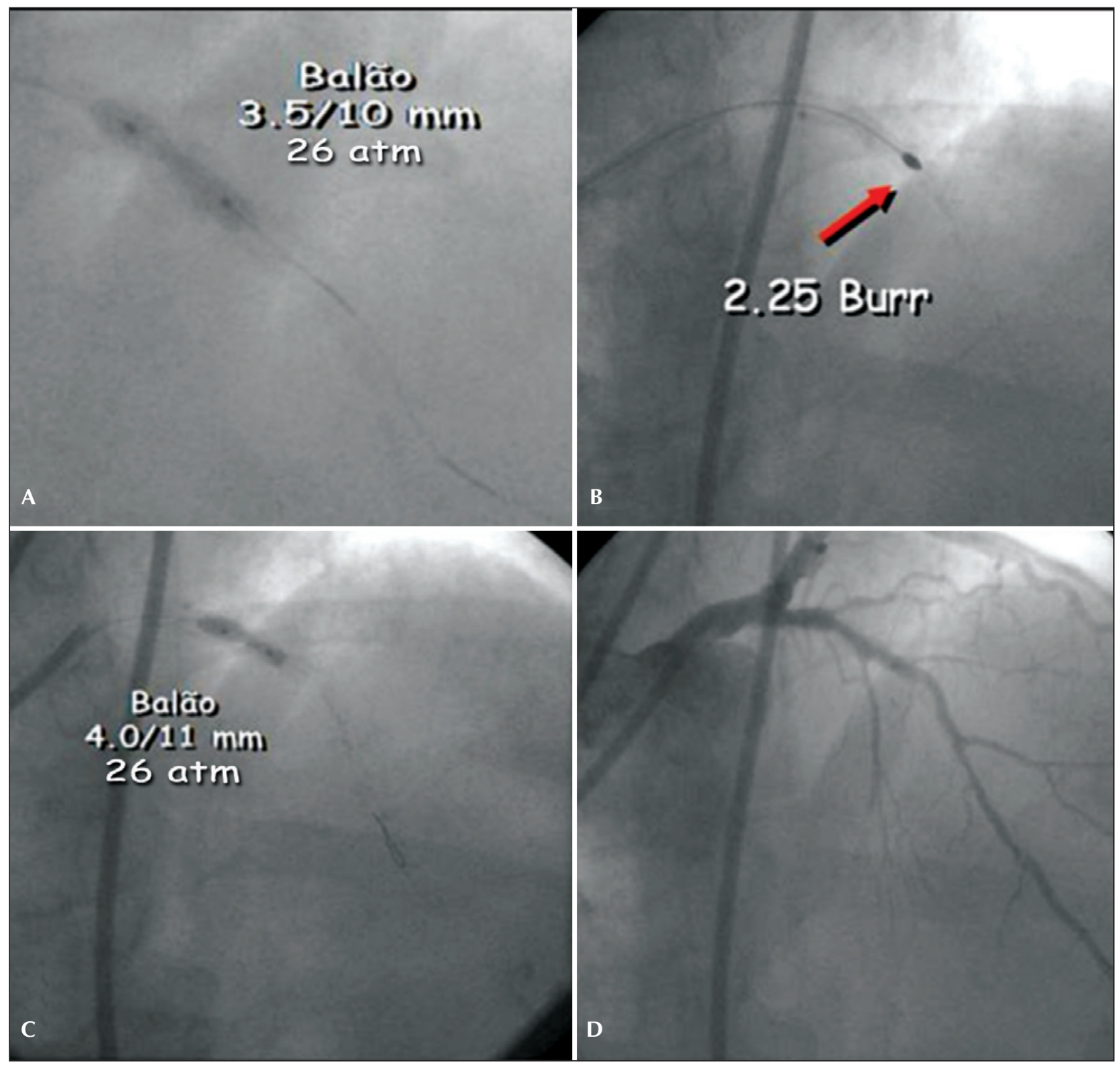

Figura 3 - Em A, angioplastia com cateter-balão $3,5 \mathrm{~mm}$ a 26 atm. Em B, aterectomia rotacional com ogiva 2,25 (seta). Em C, angioplastia com cateter-balão $4 \mathrm{~mm}$ a $26 \mathrm{~atm}$. Em D, resultado angiográfico final. 

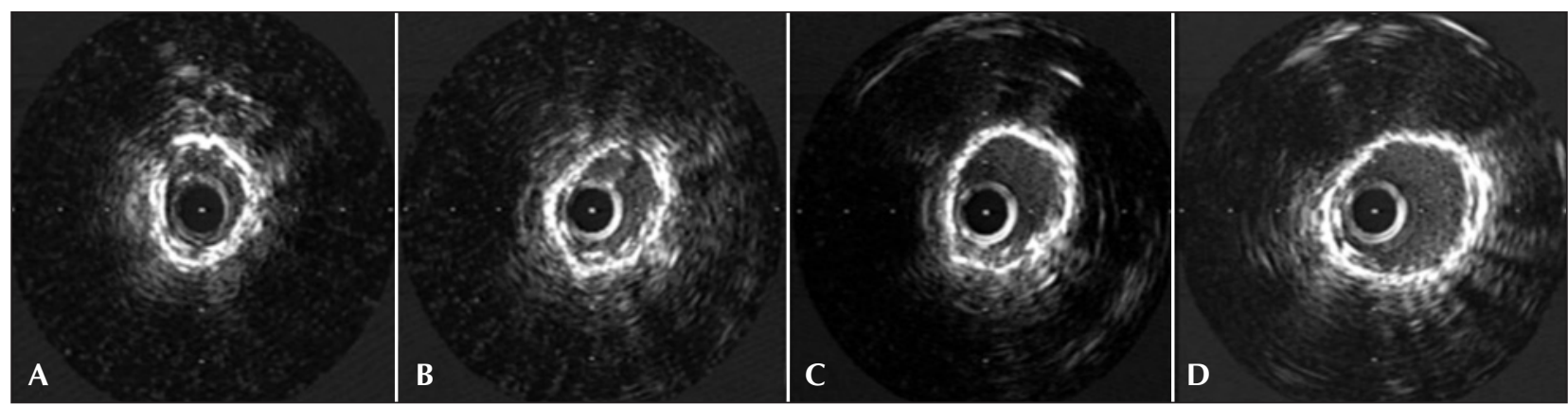

Figura 4 - Avaliação com ultrassom intracoronário no local da menor área luminal: A) hipoexpansão do stent e arco de cálcio de 360 graus pré-intervenção; B) área da luz do vaso pouco alterada pós-angioplastia com cateter-balão 3,5 mm a 26 atm; C) resultado pós-aterectomia rotacional com ogiva 2,25; e D) expansão do stent e da luz do vaso pós-angioplastia com cateter-balão $4 \mathrm{~mm}$ a 26 atm.

dado adicional de evitar quedas da velocidade de rotação < $5.000 \mathrm{rpm}$ para minimizar o número e o tamanho das partículas geradas pela ablação ${ }^{7}$.

O mecanismo de atuação da aterectomia rotacional, quando realizada dentro de stent hipoexpandido, está relacionado tanto à ablação das hastes do stent e do tecido justaposto ao mesmo, como à modificação da placa calcificada subjacente. As vibrações geradas pela ogiva podem ser transmitidas através das hastes do stent para a parede vascular, gerando microfraturas, que podem facilitar a fratura posterior do tecido calcificado com cateter-balão a alta pressão ${ }^{7}$. Em nosso caso, a aterectomia rotacional com técnica apropriada e a posterior insuflação com balão permitiram expansão da prótese e ganho luminal adequados.

É importante ressaltar que não foi constatado aumento enzimático após o procedimento, o que de certa forma afasta a hipótese, nesse caso, de embolização de partículas de cálcio ou metálicas que causassem obstrução ao fluxo coronário e subsequente necrose miocárdica.

O excimer laser também já foi utilizado como alternativa para o tratamento de reestenoses intrastent consequentes à expansão inadequada por placas muito calcificadas. Seu mecanismo de ação está associado a trauma acústico-mecânico secundário à formação de vapor por microbolhas. Na literatura, existem somente relatos da aplicação dessas técnicas em casos isolados, ficando sua utilização condicionada à apresentação desses eventos, pouco frequentes, e à disponibilidade dos instrumentais em cada centro.

\section{CONCLUSÃO}

O ulltrassom intracoronário é importante ferramenta na avaliação diagnóstica do mecanismo da reestenose intrastent, permitindo escolher a melhor estratégia para seu tratamento. A aterectomia rotacional intrastent mostrou ser uma opção para o tratamento da reestenose intrastent secundária a hipoexpansão do stent, pela presença de calcificação grave da placa aterosclerótica. A aterectomia rotacional requer experiência com o método e cuidadosa escolha das lesões a serem tratadas.

\section{CONFLITO DE INTERESSES}

Os autores declararam inexistência de conflito de interesses relacionado a este manuscrito.

\section{REFERÊNCIAS BIBLIOGRÁFICAS}

1. Forrester JS, Fishbein M, Helfant R, Fagin J. A paradigm for restenosis based on cell biology: clues for the development of new preventive therapies. J Am Coll Cardiol. 1991;17(3): 758-69.

2. Castagna MT, Mintz GS, Leiboff BO, Ahmed JM, Mehran R, Satler LF, et al. The contribution of "mechanical" problems to in-stent restenosis: An Intravascular Ultrasonographic analysis of 1090 consecutive in-stent restenosis lesions. Am Heart J. $2001 ; 142(6): 970-4$.

3. Mehran R, Dangas G, Abizaid AS, Mintz GS, Lansky AJ, Satler LF, et al. Angiographic patterns of in-stent restenosis: classification and implications for long-term outcome. Circulation. 1999;100(18):1872-8.

4. Frink RJ, Achor RW, Braun AL Jr, Kincaid OW, Brandenburg RO. Significance of calcification of the coronary arteries. Am J Cardiol. 1970;26(3):241-7.

5. Hoffmann R, Mintz GS, Popma JJ, Satler LF, Kent KM, Pichard AD, et al. Treatment of calcified coronary lesions with Palmaz-Schatz stents. An intravascular ultrasound study. Eur Heart J. 1998;19(8):1224-31. Comment in: Eur Heart J. 1998;19(8):1127-9.

6. Noble S, Bilodeau L. High energy excimer laser to treat coronary in-stent restenosis in an underexpanded stent. Catheter Cardiovasc Interv. 2008;71(6):803-7.

7. Kobayashi $\mathrm{Y}$, Teirstein $\mathrm{P}$, Linnemeier T, Stone G, Leon M, Moses J. Rotational atherectomy (stentablation) in a lesion with stent underexpansion due to heavily calcified plaque. Catheter Cardiovasc Interv. 2001 Feb;52(2):208-11. 